\title{
Efectos de la Generación y Almacenamiento Distribuidos de Energía Eléctrica en Sistemas Energéticos Regionales: análisis con el modelo LEAP
}

\section{Effects of Distributed Electric Power Generation and Storage on Regional Energy Systems: a LEAP model analysis}

Presentación: 6-7/10/2020

\section{Doctorando:}

\section{Andrés Osvaldo Benito}

Grupo CLIOPE, Facultad Regional Mendoza, Universidad Tecnológica Nacional - Argentina andresbenito@frm.utn.edu.ar

\section{Director:}

\section{Alejandro Pablo Arena}

\section{Resumen}

En este trabajo, se simula mediante del modelo LEAP (Long-range Energy Alternatives Planning), a nivel regional y a largo plazo, el rol del almacenamiento de electricidad como herramienta de flexibilidad tendiente a mejorar la paulatina integración de fuentes renovables de energía variables al sistema eléctrico de la provincia de Mendoza; entre otros, consideramos los sistemas de generación centralizados y distribuidos con tecnología fotovoltaica, teniendo en cuenta la variabilidad temporal de la fuente. Se incluye el almacenamiento de energía diario vinculado a los vehículos eléctricos (con baterías de ion-litio) en su concepción de inyección a la red (V2G). En los resultados alcanzados se observa que, con las tasas de incorporación, tanto de vehículos eléctricos, como de instalaciones fotovoltaicas distribuidas, no se aprecian variaciones considerables por su inserción al sistema eléctrico. Por el contrario, se mejoran los indicadores medioambientales. Estos hallazgos sugieren la necesidad de abordar un enfoque de optimización.

Palabras clave: energía, prospectiva, generación distribuida, almacenamiento de energía, tecnología fotovoltaica

\footnotetext{
Abstract

In this work, the role of electricity storage as a flexibility tool aimed at improving the gradual integration of variable renewable energy sources into the system is simulated, at a regional and long-term level, by means of the LEAP (Longrange Energy Alternatives Planning) model; among others, we consider centralized and distributed generation systems with photovoltaic technology of Mendoza province, taking into account the temporal variability of the source. The daily energy storage linked to electric vehicles (with lithium-ion batteries) is included in their conception of injection to the grid (V2G). In the results obtained, it is observed that, with the incorporation rates, both for electric vehicles and distributed photovoltaic systems, there are no significant variations due to their insertion into the electricity system. On the contrary, environmental indicators are improved. These findings suggest the need to address an optimization approach.
} 


\section{Introducción}

La búsqueda de un desarrollo energético sustentable es la razón de ser de la planificación energética, entendiendo que el resultado de ésta es alcanzar un plan que tenga los beneficios necesarios para guiar las actividades y recursos de un sistema energético, especialmente durante tiempos de elevada incertidumbre, entendiendo dicho sistema como el conjunto de actividades mediante las cuales las distintas fuentes de energía se producen, transportan, transforman, distribuyen y consumen para satisfacer determinadas necesidades energéticas de los sectores socio-económicos a los que está ligado dicho sistema.

Haciendo foco sólo en los requerimientos de energía eléctrica y como meta constante la búsqueda de la satisfacción de aquellos requerimientos, diversas son las posibilidades que permiten una mejor integración de generación de energía a partir de Fuentes Renovables de Energía al sector energético, tanto en su concepción concentrada como distribuida, tomando al mismo tiempo en consideración, su variabilidad dependiente de las condiciones geográficas, estacionales y climáticas (Grossi Gallegos, Hugo; Righini, 2007); (Gallegos, 1998). En este sentido, el almacenamiento de energía en forma de electricidad puede representar un modo de acumular energía cuando ésta está disponible en condiciones técnico-económicas convenientes (por ejemplo, ante una diferencia de precios), para utilizarla nuevamente cuando sea necesaria. El almacenamiento de energía eléctrica también tiene el potencial de mejorar la eficiencia y confiabilidad de la red, optimizando los flujos de energía y complementando la generación de energía a partir de fuentes renovables.

He centrado el desarrollo de este artículo en el rol que juega el almacenamiento de energía en la construcción de escenarios a largo plazo (análisis prospectivo) a nivel regional, ya que este particular no se ha estudiado en profundidad hasta ahora. Particularmente, elegimos concentrarnos sólo en el uso de las tecnologías de baterías de ionlitio asociadas al alto potencial de desarrollo que tienen de la mano del cada vez mayor uso y difusión de los vehículos eléctricos.

Para realizar el análisis conjunto y mutuamente dependiente de lo anteriormente mencionado, deben utilizarse modelos computacionales, los cuales son herramientas desarrolladas para analizar los sistemas energéticos de forma integral. En esta línea, diversos modelos han sido desarrollados con propósitos particulares(Gargiulo \& Gallachóir, 2013), así, algunos de ellos son adecuados para analizar sólo una porción del sistema y otros para analizar su totalidad (Suganthi \& Samuel, 2012); (Keirstead, Jennings, \& Sivakumar, 2012). Otro aspecto relevante, es que el análisis prospectivo suele realizarse a nivel nacional, por ende, este ejercicio raramente se lleva a cabo en niveles geográficos inferiores como las provincias de un país, sino que éstas sólo se limitan a adherirse en mayor o menor medida a las políticas estatales del país del que forman parte.

El objetivo es lograr una representación explícita de los desafíos de integración de las fuentes renovables de energía y de las opciones de flexibilidad mencionadas anteriormente, al sistema energético de la provincia de Mendoza, mediante la utilización del modelo de simulación energética a largo plazo Long-range Energy Alternatives Planning (LEAP) o modelo de Planificación de Alternativas Energéticas de Largo Plazo, según su traducción al español, el cual es probablemente el más conocido y utilizado con fines de planificación en los países en vías de desarrollo (Huang, Bor, \& Peng, 2011); (McPherson \& Karney, 2014); (Emodi, Emodi, Murthy, \& Emodi, 2017); (Sadri, Ardehali, \& Amirnekooei, 2014).

\section{Breve Caracterización del Sector Eléctrico de Mendoza}

El sector Generación de Energía se modela de acuerdo a los datos oficiales reportados al 31 de diciembre del 2018 (CAMMESA, 2018), la provincia de Mendoza posee una potencia instalada total de 1.440,00 MW, los cuales se distribuyen de forma porcentual según como sigue: 41,24\% Térmica (25.99\% Ciclos Combinados, 4.14\% Turbinas a Gas, 8.33\% Turbovapor, 2.78\% Motor Diesel), 53,64\% Hidráulica No Renovable (>50 MW) y 5,13\% Hidráulica Renovable ( $<50 \mathrm{MW})$.

A continuación, se aprecia gráficamente la generación mensual del año 2018, térmica e hidroeléctrica, tanto en MWh (Figura 1a) como en términos relativos (\%) (Figura 1b). 

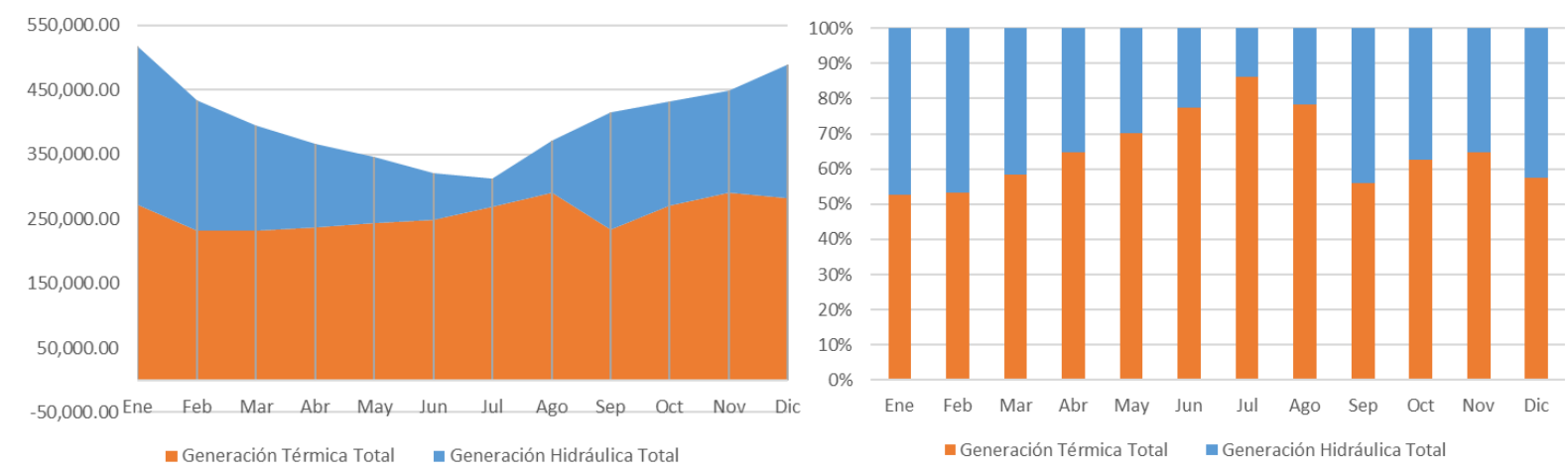

Figura 1: a) Generación Hidroeléctrica y Térmica de Mendoza. Valores Mensuales en [MWh]. Año 2018. b) Generación Hidroeléctrica y Térmica de Mendoza. Participación [\%] Mensual. Año 2018.

Al mismo tiempo, a partir de marzo del año 2015 la provincia de Mendoza, a través de su ente regulador eléctrico (EPRE, Ente Provincial Regulador Eléctrico) resolvía en su Resolución № 019/15 el Reglamento de las Condiciones Técnicas para la Operación y Facturación de Excedentes de Energía Volcados a la Red Eléctrica de Distribución. Posteriormente a nivel nacional se sanciona la ley 27.424: Régimen de Fomento a la Generación Distribuida de Energía Renovable Integrada a la Red Eléctrica Pública, la cual tiene por objeto fijar las políticas y establecer las condiciones jurídicas y contractuales para la generación de energía eléctrica de origen renovable por parte de usuarios de la red de distribución, para su autoconsumo, con eventual inyección de excedentes a la red, y establecer la obligación de los prestadores del servicio público de distribución de facilitar dicha inyección, asegurando el libre acceso a la red de distribución, sin perjuicio de las facultades propias de las provincias.

Desde el 12 de agosto de 2016, fecha en que se habilitó el primer Usuario-Generador de la provincia a la actualidad, se han incorporado anualmente como Usuarios-Generadores la cantidad de instalaciones y consecuente Potencia DC que se observan en la Figura 2 (EPRE, 2019). La simulación de los sistemas instalados, según sus características técnicas promedio, se realizó con el programa System Advisor Model Version 2018.11.11-r3 (SAM 2018.11.11-r3).

Por otra parte la caracterización del sector Demanda de energía eléctrica de la provincia de Mendoza se cuantificó con la información reportada por la Dirección de Estadísticas e Investigaciones Económicas (DEIE, 2018) de la provincia de Mendoza, según el análisis del consumo de energía eléctrica según categoría de usuarios, el cual establece los siguientes tipos de usuarios con sus consumos totales de energía: Residencial (1.562.096 MW-h), Grandes Demandas (2.491.507 MW-h), General (incluye las categorías Pequeños Comercios, Pequeña Industria, Asociaciones Civiles, Servicio Sanitario, Oficial y Tarifas transitorias) (455.171 MW-h), Riego Agrícola (508.491) y Alumbrado Público (217.355 MW-h). Los datos de transporte relacionados en particular con el parque automotor de la provincia se obtienen de la información reportada por el Observatorio Nacional de Datos de Transporte (ONDaT, 2018), del Centro Tecnológico de Transporte, Tránsito y Seguridad Vial, perteneciente a la Universidad Tecnológica Nacional, Facultad Regional Avellaneda, el cual informa que Mendoza a finales del 2017 poseía una cantidad total de 519.972 vehículos (se excluyen vehículos utilitarios livianos, vehículos de carga y autobuses). Luego, de acuerdo a la evolución del parque de automóviles de los últimos 12 años y a la variación inter-anual en dicho periodo, podemos estimar que el año 2018 finalizó con una cantidad total de 534.258 vehículos y una variación en alza de 2,75\% respecto del 2017.
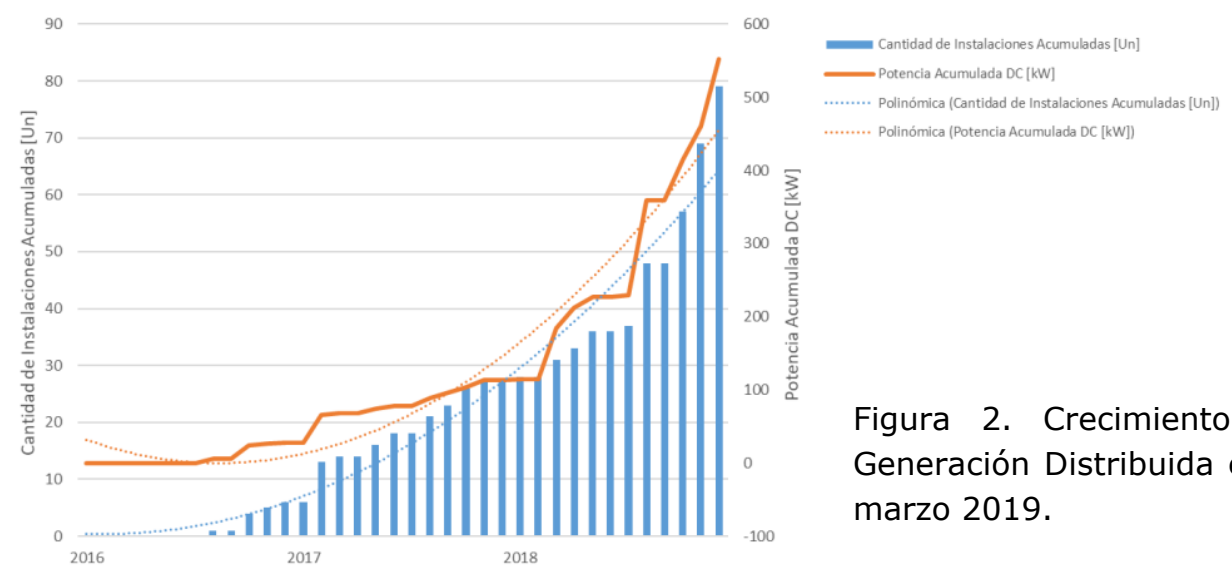

Figura 2. Crecimiento Acumulado de Instalaciones de Generación Distribuida en Mendoza. Periodo agosto 2016 marzo 2019. 


\section{Sobre el Almacenamiento de Energía Eléctrica}

En lo que respecta al almacenamiento de energía eléctrica no se puede comparar con las tecnologías de generación, ya que aporta otras importantes cualidades o valores al sistema que pueden ser clasificadas en tres aspectos: i) Valor Energético; ii) Valor de Capacidad; iii) Valor de Equilibrio, (Poudineh \& Jamasb, 2014).

\subsection{Almacenamiento Energético Distribuido}

Si bien conceptualmente podríamos considerar como almacenamiento distribuido distintas aplicaciones que nos rodean diariamente, como los teléfonos celulares personales, bicicletas y otros medios de movilidad eléctricos, en este trabajo sólo se analiza la inserción paulatina de vehículos eléctricos.

Los autos eléctricos funcionan con energía eléctrica almacenada en distintos tipos de tecnologías de medios asociadas a la fuente que luego se utilizará para suministrar la energía al vehículo, por ejemplo, medios electroquímicos como las baterías recargables (con una gran variedad de medios electroquímicos), medios químicos como las celdas de combustible o medios eléctricos como los ultracapacitores. Por lo antedicho, dependiendo de la fuente de energía, los vehículos eléctricos son de varios tipos, como vehículos eléctricos híbridos, con baterías, fotovoltaicos y con celdas de combustibles, entre otros (Chan CC, Chau KT, 2002), (US Department of Energy, 2003). Sin embargo, los vehículos eléctricos deben cargarse o recargarse ${ }^{1}$ desde la red eléctrica, lo que conlleva una demanda adicional de energía, lo que se supone un nuevo desafío para las redes eléctricas convencionales.

Tal es así, que el uso generalizado de vehículos eléctricos puede causar posibles problemas técnicos: por ejemplo, la red eléctrica puede verse perjudicada por eventos de recarga no controlados y por recargas excesivamente prolongadas en el tiempo. Además, la Demanda Agregada de Carga/Recarga debe controlarse cuidadosamente para evitar la interrupción del servicio eléctrico cuando se introduzcan al sistema varios miles de vehículos eléctricos en un corto periodo de tiempo, como puede suceder en estacionamientos públicos o estaciones de recarga (Yagcitekin, Uzunoglu, Karakas, \& Erdinc, 2015).

\section{Metodología}

En nuestro marco de trabajo, el sistema de simulación LEAP (Long-range Energy Alternatives Planning) se utiliza como una herramienta de modelado de contabilidad energética que iguala la demanda con la generación de energía eléctrica del lado de la oferta, describiendo los impactos del sistema, incluyendo la generación de electricidad por fuente y el potencial de emisión de gases de efecto invernadero (GEI). Sobre la base del sector eléctrico de Mendoza, se desarrollan tres escenarios, para los cuales el año 2018 se utiliza como año base para todos los senderos energéticos.

El escenario BAU o Tendencial (Bussiness As Usual por sus siglas en inglés) es un sendero energético centrado en las políticas y planes actuales de la Secretaría de Energía, dependiente del Ministerio de Hacienda de la Nación, de la Compañía Administradora del Mercado Mayorista Eléctrico S.A. (CAMMESA) y a nivel provincial de la Empresa Mendocina de Energía SAPEM (EMESA, 2018). Luego el escenario REN-GD se centra en la incorporación de los parques fotovoltaicos y pequeñas centrales hidroeléctricas (PCHs) adjudicados en la edición 1.5 y 2.0 del Programa RenovAr: Al mismo tiempo, se contemplan las proyecciones de inserción de generación distribuida a partir de tecnología fotovoltaica. Por su parte, el escenario V2G se focaliza en las proyecciones de sustitución de automóviles a combustible fósil por automóviles eléctricos y cómo ésta tecnología puede ser utilizada como una herramienta de almacenamiento distribuido que permita equilibrar las intermitencias natas que presenta la generación a partir de fuentes variables de energía renovable.

En el modelo se dejan establecidos los parámetros técnicos necesarios para la simulación tales como: intensidades energéticas para la demanda y reglas de despacho, órdenes de mérito, eficiencia de los procesos, producciones históricas, capacidades, disponibilidades máximas, curca de duración de carga, partición anual, entre otros para el sector generación. También se especifica una base de datos de tecnología y medio ambiente que incluye los factores de emisión que establecen valores para las emisiones de GEI, asociados con la combustión de los diferentes combustibles en la generación de electricidad.

${ }^{1}$ El término "carga" o "recarga" de vehículos eléctricos se utiliza indistintamente para hacer referencia a la situación en la que el vehículo absorbe energía desde una red eléctrica. 


\section{Resultados}

Se observa en la Figura 3 cómo gana participación la generación de energía a partir de centrales fotovoltaicas en su configuración centralizada, al mismo tiempo que la generación de centrales hidroeléctricas también es preponderante, ambas en detrimento de generación con base en combustibles fósiles. La curva de incorporación de generación acompaña la forma del crecimiento de la demanda que se observa en la Figura 4.

Adicionalmente podemos decir, de forma preliminar, que, con las tasas de incorporación de vehículos eléctricos planteadas, no se aprecian variaciones considerables al considerar la inyección de energía remante en las baterías a la red de distribución. Esto implicaría que debe fomentarse aún más el uso de movilidad eléctrica.

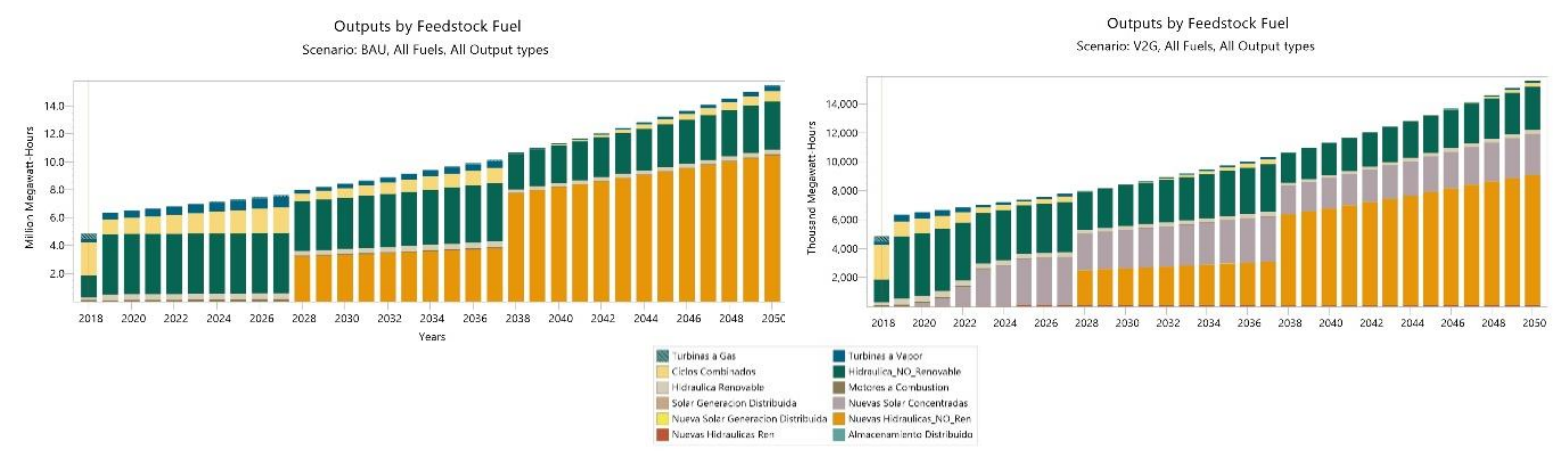

Figura 3. Generación de Energía por Fuente. Comparación entre el escenario BAU y el escenario V2G.

En la Figura 4a se aprecia el crecimiento de la demanda del sector residencial como consecuencia del crecimiento poblacional a las tasas reportadas en los últimos 10 años. De acuerdo a las hipótesis planteadas para el escenario V2G se aprecia cómo procede el retiro de vehículos que funcionan con combustibles fósiles (lo que se ve plasmado en el consumo de nafta y diésel), en pos de la inserción de vehículos eléctricos, sin embargo, la incorporación de estos últimos no presenta una tendencia al alza del consumo de energía, sin por el contrario con consumo del sector transporte vehicular continua a la baja.

Finalmente, y de modo resumido podemos decir que las emisiones de gases de efecto invernadero, mostradas bajo el indicador de Potencial de Calentamiento Global a 100 años (100-Year GWP: Direct (At Point of Emissions) se ven reducidas por la disminución del consumo de combustibles fósiles para el sector Transporte Vehicular, como consecuencia de la política de fomento del uso de vehículos eléctricos. Esto se observa en la Figura 4b.

\section{Conclusiones y Perspectivas Futuras}

La representación de la operación del sistema energético requiere tener en cuenta ciertos detalles técnicos y temporales, por ejemplo, la variabilidad de la energía solar de acuerdo a sus restricciones inter-temporales. El almacenamiento de electricidad también requiere este tipo de restricción ya debe ser equilibrado temporalmente. Estas restricciones sugieren la necesidad de abordar un enfoque de optimización.
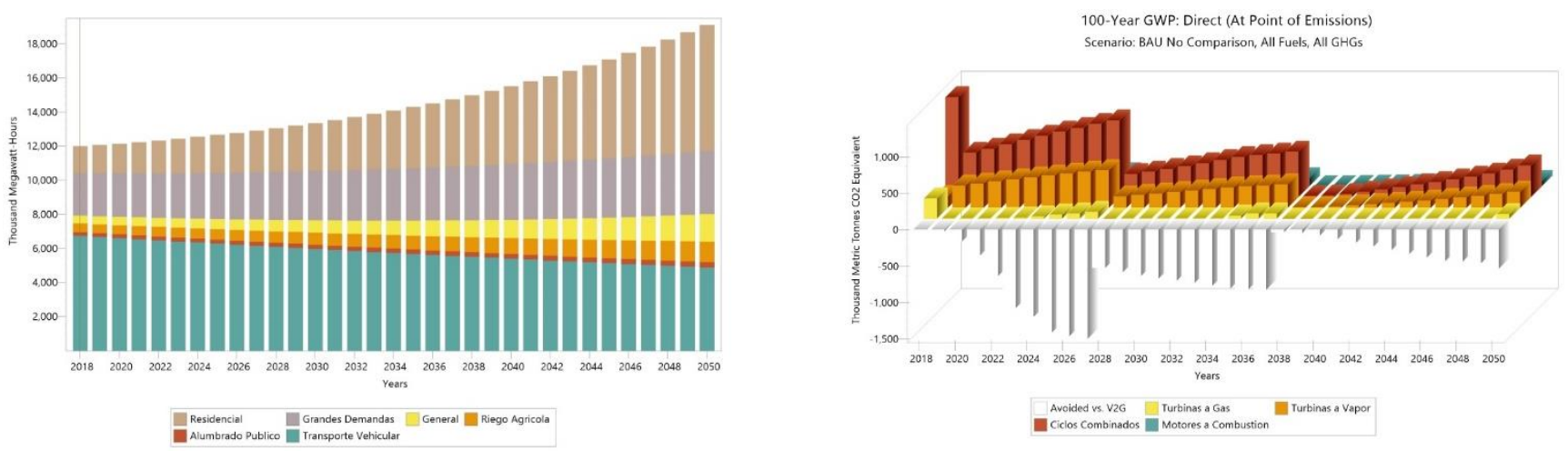

Figura 4. a) Proyección de la Demanda. Escenario V2G. Provincia de Mendoza, Rep. Argentina. Año 2018 a 2050; b) Emisiones evitadas. Comparación escenario BAU versos V2G. Indicador: 100-Year GWP: Direct (At Point of Emissions). Provincia de Mendoza, Rep. Argentina. Año 2018 a 2050. 
A su vez, la variabilidad de los recursos renovables requiere la construcción de una Curva de Duración de Carga Residual, también llamada Curva de Carga Residual o Curva de Demanda Neta, la cual se construye restando a la curva de demanda los valores de generación de las centrales e instalaciones autodespachable, es decir las centrales de energía solar, tanto concentradas como distribuidas. Por lo tanto, la Demanda Neta es la que realmente debe ser cubierta con generación convencional, con capacidad de adecuarse a las necesidades de la demanda (despachable).

Se plantea la implementación en el modelado de otros instrumentos de almacenamiento de energía, como, por ejemplo, sistemas hidráulicos a partir de los embalses de ciertas centrales hidráulicas de la provincia, de manera que estas alternativas puedan conjugarse técnicamente con el almacenamiento distribuido planteado a través de las baterías de los vehículos eléctricos de incipiente incorporación regional.

El sistema energético actual no es sostenible y debe transformarse en un sistema con bajas emisiones en una a largo plazo. Por lo tanto, algunas decisiones importantes deben tomarse ahora en el presente, justificando la necesidad de escenarios integrales y a largo plazo de todo el sistema energético regional. Se espera que el sector eléctrico pueda verse beneficiado con relativa facilidad mediante la generación y el transporte de energía electricidad de origen renovable, y que ello se presente como una herramienta crucial en pos de alcanzar un sistema energético con menor carga de emisiones de carbono. Sin embargo, un sistema energético con alta participación de energías renovables se convierte en un desafío, principalmente debido a la variabilidad de algunas de las fuentes correspondientes. Por lo tanto, los escenarios energéticos a largo plazo tienen que integrar estas características de forma cuantitativa.

\section{Referencias}

Dirección de Estadísticas e Investigaciones Económicas (DEIE), Ministerio de Economía, Infraestructura y Energía, Gobierno de Mendoza. (2018). Mendoza en Datos, Datos Económicos: i) Población estimada al 1o de julio de cada año calendario por sexo (ambos sexos), según departamento. Mendoza. Años 2010-2025; ii) Producto Bruto Geográfico. Valor Agregado Bruto por año y tasa de crecimiento interanual. Mendoza. Años 2004-2017; iii) Consumo de energía eléctrica según categoría de usuarios. Mendoza. Años 2003-2018.

Emodi, N. V., Emodi, C. C., Murthy, G. P., \& Emodi, A. S. A. (2017). Energy policy for low carbon development in Nigeria: A LEAP model application. Renewable and Sustainable Energy Reviews, 68(August 2015), 247-261. https://doi.org/10.1016/j.rser.2016.09.118

Gallegos, H. G. (1998). Distribución de la radiación solar global en la República Argentina. II. Cartas de radiación. Energías Renovables y Medio Ambiente, 5(January 1998), 33-42.

Gargiulo, M., \& Gallachóir, B. Ó. (2013). Long-term energy models: Principles, characteristics, focus, and limitations. Wiley Interdisciplinary Reviews: Energy and Environment, 2(2), 158-177. https://doi.org/10.1002/wene.62

Grossi Gallegos, Hugo; Righini, R. (2007). Atlas de energía solar de la República Argentina. In Asociación Argentina de Energías Renovables y Ambiente - ASADES.

Huang, Y., Bor, Y. J., \& Peng, C. Y. (2011). The long-term forecast of Taiwan's energy supply and demand: LEAP model application. Energy Policy, 39(11), 6790-6803. https://doi.org/10.1016/j.enpol.2010.10.023

Keirstead, J., Jennings, M., \& Sivakumar, A. (2012). A review of urban energy system models: Approaches, challenges and opportunities. Renewable and Sustainable Energy Reviews, 16(6), 3847-3866. https://doi.org/10.1016/j.rser.2012.02.047

McPherson, M., \& Karney, B. (2014). Long-term scenario alternatives and their implications: LEAP model application of Panama's electricity sector. Energy Policy, 68, 146-157. https://doi.org/10.1016/j.enpol.2014.01.028

Poudineh, R., \& Jamasb, T. (2014). Distributed generation, storage, demand response and energy efficiency as alternatives to grid capacity enhancement. Energy Policy, 67, 222-231. https://doi.org/10.1016/j.enpol.2013.11.073

Sadri, A., Ardehali, M. M., \& Amirnekooei, K. (2014). General procedure for long-term energy-environmental planning for transportation sector of developing countries with limited data based on LEAP (long-range energy alternative planning) and EnergyPLAN. Energy, 77, 831-843. https://doi.org/10.1016/j.energy.2014.09.067

Suganthi, L., \& Samuel, A. A. (2012). Energy models for demand forecasting - A review. Renewable and Sustainable Energy Reviews, 16(2), 1223-1240. https://doi.org/10.1016/j.rser.2011.08.014

Yagcitekin, B., Uzunoglu, M., Karakas, A., \& Erdinc, O. (2015). Assessment of electrically-driven vehicles in terms of emission impacts and energy requirements: A case study for Istanbul, Turkey. Journal of Cleaner Production, 96, 486-492. https://doi.org/10.1016/j.jclepro.2013.12.063 